\title{
Identification of Adhesins in Plant Beneficial Rhizobacteria Bacillus velezensis SQR9 and Their Effect on Root Colonization
}

\author{
Rong Huang, ${ }^{1}$ Haichao Feng, ${ }^{1}$ Zhihui $X u,{ }^{1,}{ }^{\dagger}$ Nan Zhang, ${ }^{1}$ Yunpeng Liu, ${ }^{2}$ Jiahui Shao, ${ }^{1}$ Qirong Shen, ${ }^{1}$ and \\ Ruifu Zhang ${ }^{2, \dagger}$ \\ ${ }^{1}$ Jiangsu Provincial Key Lab of Solid Organic Waste Utilization, Jiangsu Collaborative Innovation Center of Solid Organic \\ Wastes, Nanjing Agricultural University, Nanjing 210095, Jiangsu, P.R. China \\ ${ }^{2}$ Key Laboratory of Microbial Resources Collection and Preservation, Ministry of Agriculture, Institute of Agricultural Resources \\ and Regional Planning, Chinese Academy of Agricultural Sciences, Beijing 100081, P.R. China
}

Accepted 6 October 2021.

\begin{abstract}
Probiotic Bacillus colonization of plant root surfaces has been reported to improve its beneficial effect. Chemotaxis, adhesion, aggregation, and biofilm formation are the four steps of root colonization by plant growth-promoting rhizobacteria (PGPRs). Compared with the other three well-studied processes, adhesion of PGPRs is less known. In this study, using mutant strains deleted for potential adhesin genes in PGPR strain Bacillus velezensis SQR9, adherence to both cucumber root surface and abiotic surface by those strains was evaluated. Results showed that deletion mutations $\Delta l y t B$, $\Delta V 529 \_10500, \Delta f l i D, \Delta y h a N$, and $\Delta s a c B$ reduced the adhesion to root surfaces, while, among them, only $\Delta f l i D$ had significant defects in adhesion to abiotic surfaces (glass and polystyrene). In addition, $B$. velevzensis SQR9 mutants defective in adhesion to root surfaces showed a deficiency in rhizosphere colonization. Among the encoded proteins, FliD and YhaN played vital roles in root adhesion. This research systematically explored the potential adhesins in a well-studied PGPR strain and also indicated that adhesion progress was required for root colonization, which will help to enhance rhizosphere colonization and beneficial function of PGPRs in agricultural production.
\end{abstract}

Keywords: abiotic surface, adhesin, Bacillus velezensis SQR9, colonization, root surface

Biofilms are communities of surface-related microorganisms encased in the self-generated extracellular matrix. The formation of biofilm is a universal bacterial feature and is found on almost all natural and artificial surfaces, including solid surfaces and

${ }^{\dagger}$ Corresponding authors: Z. Xu; xzh2068@njau.edu.cn and

R. Zhang; zhangruifu@caas.cn

Funding: This research was financially supported by the National Natural Science Foundation of China (grant number 31870096).

*The $\boldsymbol{e}$-Xtra $\operatorname{logo}$ stands for "electronic extra" and indicates there are supplementary materials published online.

The author(s) declare no conflict of interest.

(c) (1) () () Copyright () 2021 The Author(s). This is an open access article distributed under the CC BY-NC-ND 4.0 International license. liquid-air interfaces (Hall-Stoodley et al. 2004; Ramey et al. 2004; Stewart and Franklin 2008). Biofilms protect bacteria from environmental stress and enhance the interaction between bacteria and various biotic or abiotic surfaces, which result in the efficient colonization of bacteria on different surfaces. Bacillus subtilis, a nonpathogenic gram-positive bacterium, has become a model microorganism for studying biofilm formation. B. subtilis can form pellicle biofilms at the liquid-air interface of standing cultures and complex colony architectures on solid surfaces (Fan et al. 2011; Xu et al. 2014). The biofilm formation process includes several stages of development, maturation, and disassembly (Vlamakis et al. 2013). At the start of biofilm formation, motile cells with flagella are differentiated into nonmotile matrix-producing cells, which stop separating and form the extracellular matrix surrounded chains. In a mature biofilm, the cells that produce the matrix are sporulated. In aged biofilms, some cells secrete small molecules such as D-amino acids and polyamines to break down the extracellular matrix, allowing the cells to disperse in the environment (Vlamakis et al. 2013). The early stages of biofilm formation consist of chemotaxis, adhesion, aggregation, and biofilm formation (Aguilar et al. 2007; Smit et al. 1989).

Attachment to surfaces is the initial step for biofilm formation (Merritt et al. 2007). For example, the association of Herbaspirillum seropedicae with poaceous crops was initiated with the adhesion of the bacteria to root surfaces, followed by colonization at the emergence points of secondary roots (Balsanelli et al. 2010). Azospirillum brasilense is a plant growth-promoting rhizobacterium (PGPR); adhesion played an important role in its colonization on wheat root (Croes et al. 1993). Among factors of persistent infection in plants by Xylella fastidiosa, adhesion played a partial role in the initial stages of biofilm formation (de Souza et al. 2005).

The adhesion ability of microbes depends on their surface appendages anchored in the bacterial outer membrane taking part in attachment to one or both abiotic and biotic surfaces (Moreira et al. 2004). Flagella, the protrudent and filamentous appendages of some bacteria, participate in the adhesion process. Salmonella spp. attach to cholesterol through FliC, a flagellum protein, as the initial step for colonizing and forming biofilms on human gall stones (Crawford et al. 2010). The lipopolysaccharide (LPS) of gram-negative bacteria had an effect on cell adhesion. The lack of B-band LPS in Pseudomonas aeruginosa PAO1 demonstrated reduced adhesion to hydrophilic surfaces (Makin and Beveridge 1996). LPS-lacking Escherichia coli also 
decreased the adhesive ability (Genevaux et al. 1999). Herbaspirillum seropedicae needed LPS to attach the maize root (Hori and Matsumoto 2010; Nevola et al. 1985). Collagen like proteins (CLPs) are located in the outer layer of bacterial cells, which are important for bacterial adhesion to host cells and biofilm formation. CLPs of Bacillus amyloliquefaciens FZB42 appeared to play a role in bacterial aggregation and adherence to the surfaces of abiotic materials and Arabidopsis roots (Zhao et al. 2015). Some polysaccharide capsules surrounding the bacteria can interact with legumes, because specific carbohydrate structures in the polysaccharide capsule can be recognized by lectin molecules produced from legume root hair tips. In Rhizobium leguminosarum RBL5523, there might exist two different lectin ligands, one for the clover root and the other for the pea/ vetch root (Laus et al. 2004, 2006). Cell-wall hydrolases (CWHs) are surface enzymes to degrade cell wall by cleaving specific covalent bonds (López and García 2004). LytB is a $\mathrm{CWH}$ of Streptococcus pneumoniae and participates in the initial attachment to the nasopharynx; effective colonization of the respiratory tract needs the combination of CWHs LytB and LytC (Ramos-Sevillano et al. 2011).

Bacillus species are model microorganisms for biofilm study. The $B$. cereus spores have long appendages playing important roles in the surface attachment (Faille et al. 2010; Klavenes et al. 2002). The exosporium glycoprotein BclA filaments played a major role in interaction of spores with stainless steel by providing a larger contact surface (Lequette et al. 2011). S-layer components, flagellin, and cell-bound proteases of B. cereus have been found to bind intestinal surface components such as mucin or fibronectin (Sánchez et al. 2009). Cell-wall peptidase CwpFM of B. cereus was implicated in adhesion to epithelial cells (Tran et al. 2010). In B. anthracis, BslA (S-layer protein A) was sufficient for adhesion of vegetative cells to human endothelial cells (Tonry et al. 2012), however, overexpression of the pleiotropic regulator CodY in B. anthracis decreased the attachment to sterile polystyrene (Gopalani et al. 2016). Cell-surface proteins played an important role in the initial process of attachment to stainless steel of the vegetative cells and spores of thermophilic Bacilli spp. (Parkar et al. 2001). The spore exosporium of $B$. mycoides has been observed to be flexible and to promote adhesion to glass surface of the spore by increasing the spore contact area on the surface (Bowen et al. 2002). Adhesion to gold surfaces by B. subtilis NCIB 3610 was mediated by thiol groups present in proteins of the bacterial cell membrane (Kesel et al. 2014). Filamentous hemagglutinin adhesin is a large filamentous protein, which was required in B. licheniformis adherence to microtiter tissue culture plate (Sadiq et al. 2019). Bacillus species are representative PGPRs; root colonization is a prerequisite for PGPRs to perform their plant beneficial functions. Biofilm formation of Bacillus on rhizoplane is well studied, but the root attachment process is less known.

In this study, the potential adhesins contributed to root attachment of a well-studied PGPR strain, Bacillus velezensis SQR9 (formerly Bacillus amyloliquefaciens SQR9), were systematically explored. B. velezensis SQR9 was isolated from the cucumber rhizosphere. As a strong root colonizer, SQR9 formed robust and highly structured biofilms and efficiently promoted plant growth and suppressed soil-borne diseases (Qiu et al. 2014; Weng et al. 2013). The complicated regulatory network governing $B$. velezensis SQR9 rhizosphere chemotaxis and biofilm formation has been well-elucidated (Dong et al. 2018; Feng et al. 2018; Li et al. 2018; Xu et al. 2013, 2014, 2019; Zhou et al. 2018). Here, we show that $B$. velezensis SQR9 has multiple adhesin proteins for root and abiotic surface attachment, which are required for its efficient rhizoplane colonization. These results provide guidelines for enhancing the rhizosphere colonization and beneficial function of PGPR in agricultural production.

\section{RESULTS}

Prediction of potential adhesin proteins in $B$. velezensis SQR9 and construction of adhesin mutants and corresponding complementation strains.

The entire genome of $B$. velezensis $\mathrm{SQR} 9$ has been sequenced, and a gene annotation table has been made by our laboratory that contains results from Kyoto Encyclopedia of Genes and Genomes orthology, National Center for Biotechnology Information (NCBI) (accession number CP006890), and Pfam databases. To systematically explore possible adhesins in B. velezensis SQR9, we searched the annotation table for reported adhesins and possible adhesin-related proteins. Seven possible adhesin-related proteins have been identified. LytB (Ramos-Sevillano et al. 2011) and FliD (Tasteyre et al. 2001) are proteins that have been reported to be involved in adhesion. $\mathrm{SacB}$ is a levansucrase responsible for the synthesis of levan (Pereira et al. 2001), which is a polysaccharide that may be involved in adhesion (Manandhar et al. 2009). V529_10500 and YcdA are membrane lipoproteins, playing a role in cell adhesion (Knoke et al. 2020). V529_07150 contains a collagen-binding domain, which exhibits the best surface complementarity to the collagen, and the domain can be found in a Staphylococcus aureus adhesin (Symersky et al. 1997). These seven proteins were predicted by another database, SMART, and possible domains of each protein are listed in Table 1 . These genes were deleted individually in SQR9 to obtain their mutants $(\Delta l y t B$, $\Delta V 529 \_10500, \Delta y c d A, \Delta V 529 \_07150, \Delta f l D, \Delta y h a N$, and $\left.\Delta s a c B\right)$ as well as their corresponding complementation strains $(\Delta l y t B /$ lytB, DV529_10500/V529_10500,_tfiD/fliD, syhaN/yhaN, and $\Delta s a c B / s a c B)$. Protein YcdA and V529_07150 did not show root surface adhesion function and the deleted genes were not complemented in the following research (Supplementary Table S1).

\section{Contribution of potential adhesins}

to the root attachment of $B$. velezensis SQR9.

Initial attachment to the rhizoplane is an important step for PGPRs to colonize the root and perform plant-beneficial functions; bacterial adhesins and their interactions with plant roots play important roles during the attachment process. Root attachment analysis revealed that these potential adhesin genes (except for $y c d A$ and V529_07150) significantly affected SQR9 root attachment $(n=5, P<0.05)$. Compared with the wild-type strain, different mutants showed 20 to $50 \%$ reduced root attachment (Fig. 1). Mutants $\triangle y c d A$ and $\Delta V 529 \_07150$ showed the same adhesion ability with the wild-type strain while other mutants decreased this ability to root surfaces.

Gene complementation of these root attachment-defective mutants ( $\Delta l y t B / l y t B, \Delta V 529 \_10500 / V 529 \_10500, \Delta y h a N / y h a N$, and $\Delta s a c B / s a c B)$ partially restored the impaired attachment function (Fig. 1). Confocal laser scanning microscopy (CLSM) of the cucumber root segments attached by different strains of SQR9 confirmed these observations. Although only a small number of cells were detected on the root surface, wild-type and genecomplementation strains showed more fluorescent dots compared with the corresponding mutants (Fig. 2). The combination of quantitative and qualitative results validated the adhesion function of these candidate genes in the PGPR strain B. velezensis SQR9, especially, $\Delta y h a N / y h a N$ greatly increased adhesion ability to cucumber root surfaces compared with $\Delta y h a N$.

\section{Contribution of adhesins to the abiotic surface attachment of $B$. velezensis SQR9.}

To test if the surface attachment of these adhesins in B. velezensis SQR9 are specific to biotic material of root, attachment assays to glass and polystyrene surfaces were also performed. 
The fliD mutant had a defect in attachment ability on abiotic surfaces, its complementation strain did not restore this ability. The adhesion ability of other mutants ( $\Delta l y t B, \Delta V 529 \_10500, \Delta y h a N$, and $\Delta s a c B$ ) to glass and polystyrene surfaces were similar to the wild type, and their complementation strains showed almost the same ability as the mutants (except for the complementation strain $\Delta V 529 \_10500 / V 529 \_10500$, whose adhesion ability on polystyrene surfaces was still defective) (Fig. 3). Thus, most of these adhesins did not take part in the attachment to abiotic surfaces. CLSM scanning of these plastic and glass surfaces attached by different strains of SQR9 revealed that there were no significant differences between the mutants and the wild type or between the mutants and their complementation strains (Supplementary Figs. S4 and S5).

\section{Effect of adhesins on chemotaxis and biofilm formation of $B$. velezensis SQR9.}

Chemotaxis is an important step of bacterial attachment (Pratt and Kolter 1998). We assessed the effect of these potential adhesins on the SQR9 chemotactic response to the known chemoattractant malic acid. Results showed that only fliD gene deletion affected SQR9 chemotaxis, since the motility was impaired (Supplementary Fig. S1). In addition, the biofilm formation was also tested, none of the mutant strains showed colony architecture (aggregation ability) deficiency on a solid minimal salts glutamate glycerol (MSgg) and lysogeny broth (LB) agar plate (Supplementary Fig. S2). Mutant strains could form pellicle biofilms in the air-liquid interface of liquid MSgg medium, except for $\Delta f i D$ (Supplementary Fig. S3).

\section{Effect of adhesins to the rhizosphere colonization of B. velezensis SQR9.}

Adhesion is the early step for rhizoplane colonization of PGPRs. We then investigated if these adhesins in B. velezensis SQR9 affect its root colonization. After coculturing strains and cucumber roots for $24 \mathrm{~h}$, the number of colonization cells was counted. Compared with the wild-type strain SQR9, mutants
$\Delta l y t B, \Delta y h a N$, and $\Delta s a c B$ showed significantly reduced colonization ability, while the root colonization of other mutants and all complementation strains had no significant differences with the wild-type strain SQR9 (Fig. 4). For glass and plastic surfaces, only $\triangle$ fliD showed obviously decreased colonization, which can be restored by $f i D$ gene complementation, probably due to the effect of motility on the colonization (Supplementary Fig. S6).

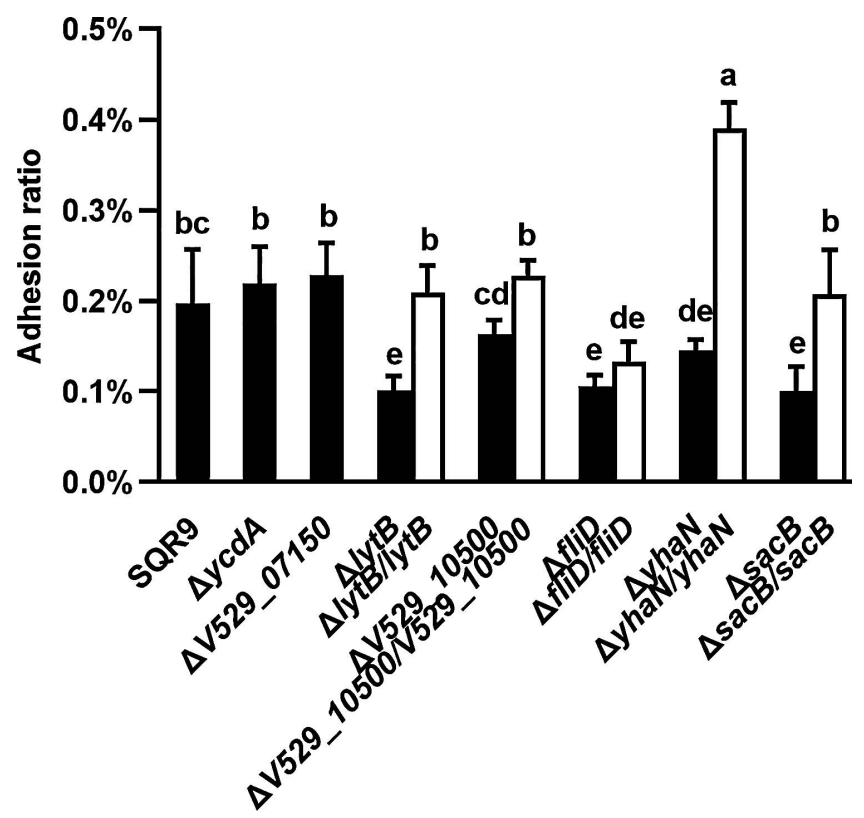

Fig. 1. Adherence ability of Bacillus velezensis SQR9 and derived mutants or complementation strains to cucumber roots. The adherence ability was indicated by adhesion ratio, i.e., the number of bacteria attached to cucumber roots relative to inoculation amount. Error bars are standard deviation, $n=5$. Different letters mean significant differences $(P<0.05$, one-way analysis of variance).

Table 1. Potential adhesin-like proteins of Bacillus velezensis SQR9 and their structural and functional analysis

\section{Possible adhesin-like}

proteins

LytB

V529_10500

YcdA

V529_07150

FliD

YhaN

$\mathrm{SacB}$
Structures $^{\mathbf{a}}$

LytB contains an OmpH-like domain (67-246 aa), which interacts directly with membrane lipids and lipopolysaccharide (Walton and Sousa 2004) as well as a bacterial Ig-like domain (312-391 aa), which is present in a bacterial adhesin molecule (Kelly et al. 1999)

V529_10500 contains subunits (44-323 aa) of integrin, which is a cell adhesin molecule (Bökel and Brown 2002)

YcdA contains an OmpH-like domain (9-228 aa) and a bacterial Ig-like domain (33-126 aa)

V529_07150 contains a bacterial Ig-like domain (370-443 aa) and a dystroglycan-type cadherin-like domain $(1,102-1,197$ aa) that is present in adhesin molecules (Dickens et al. 2002)

FliD contains a dystroglycan-type cadherin-like domain (48-135 aa), a bacterial Ig-like domain (197-275 aa), and the domain of formin homology (19-475 aa), which participates in the reorganization of the actin cytoskeleton that mediates adhesion (Tanaka 2000)

YhaN includes an OmpH-like domain (126-235 aa) and a formin homology domain (511-898 aa)

SacB contains a glyco domain that belongs to the glycosyl hydrolases family (56-464 aa)
Functions

An enhancer protein of amidase, the cell wall hydrolase; a modifier protein of major autolysin LytC (Lazarevic et al. 1992; Ramos-Sevillano et al. 2011)

An ABC transporter substrate-binding protein (Mistry et al. 2021)

A putative lipoprotein that falls into the antigen superfamily (Mistry et al. 2021)

A collagen binding domain-containing protein (Mistry et al. 2021)

A flagellar capping protein involved in flagellin assembly (Tasteyre et al. 2001)

A putative $\mathrm{ABC}$ transporter (National Center for Biotechnology Information database)

A levansucrase that belongs to the glycosyl hydrolase family (Pereira et al. 2001)

${ }^{a}$ The structure analysis of the seven predicated adhesin-like proteins was based on the SMART database. The position of each domain is enclosed in brackets. 
Comparisons of adhesin genes in plant-associated and non-plant associated Bacillus strains.

To further investigate if these adhesins are related to the differentiation of plant-associated (PA) and non-plant associated (non-PA) Bacillus strains, 16 strains of $B$. amyloliquefaciens or B. velezensis (Zhang et al. 2016), which are closely related to SQR9 and of which 12 strains are PA and four strains are nonPA, were compared with SQR9 for these five adhesin genes. In addition, the whole-genome average nucleotide identity (ANI) values of SQR9 and these Bacillus strains were calculated to exclude the effect of phylogenetic relationship on the comparison of these adhesin genes.
As shown in Table 2, the lytB and V529_10500 genes in SQR9 have low identities with homologous genes in non-PA Bacillus strains (67 and 84\%, respectively) but high identity (>97\%) with PA strains. Thus, lytB and V529_10500 are conserved in PA Bacillus. Gene yhaN in SQR9 has $92 \%$ identity with the homologous gene in non-PA Bacillus strains, which is slightly lower than the ANI (93\%), while it has high identity (98 to $99 \%$ ) with its homologous gene in PA Bacillus strains, suggesting that yhaN might be conserved in PA Bacillus strains. Gene $f i D$ in SQR9 has $92 \%$ identity with its homologous gene in non-PA Bacillus strains, which is slightly lower than the ANI (93\%); but it also has low identity (94\%) with the homologous
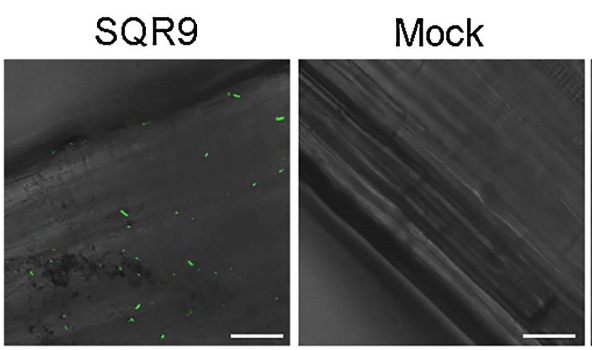

$\triangle$ fil

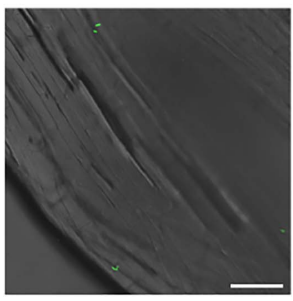

$\triangle$ fliD/fIID

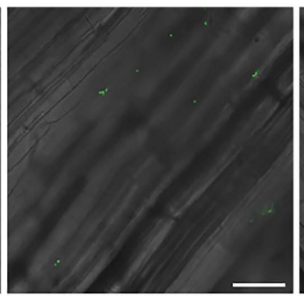

$\Delta y t B$

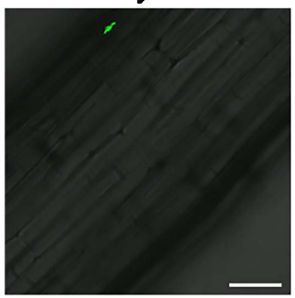

$\triangle \operatorname{sacB}$

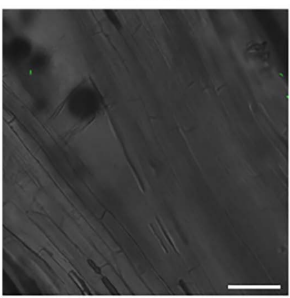

$\Delta l y t B / y t B$

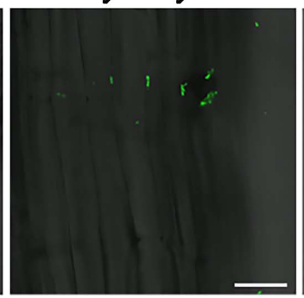

$\triangle \mathrm{sacB} / \mathrm{sacB}$

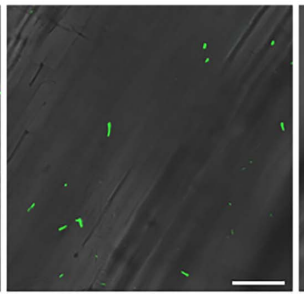

$\triangle V 529 \quad 10500 /$
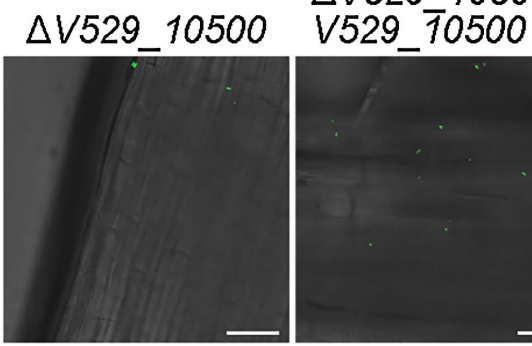

$\triangle y h a N$
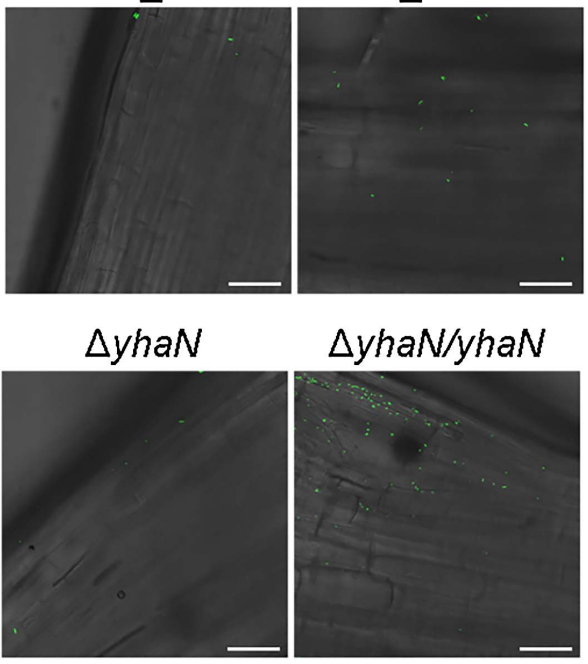

Fig. 2. Confocal laser scanning micrographs of the adhesion of green fluorescent protein-tagged Bacillus velezensis SQR9, mutants, and complementation strains on the roots. The root samples were taken $30 \mathrm{~min}$ after inoculation. Mock represents the cucumber roots without bacterial inoculation. The results are representative of three experiments. Bar $=50 \mu \mathrm{m}$.
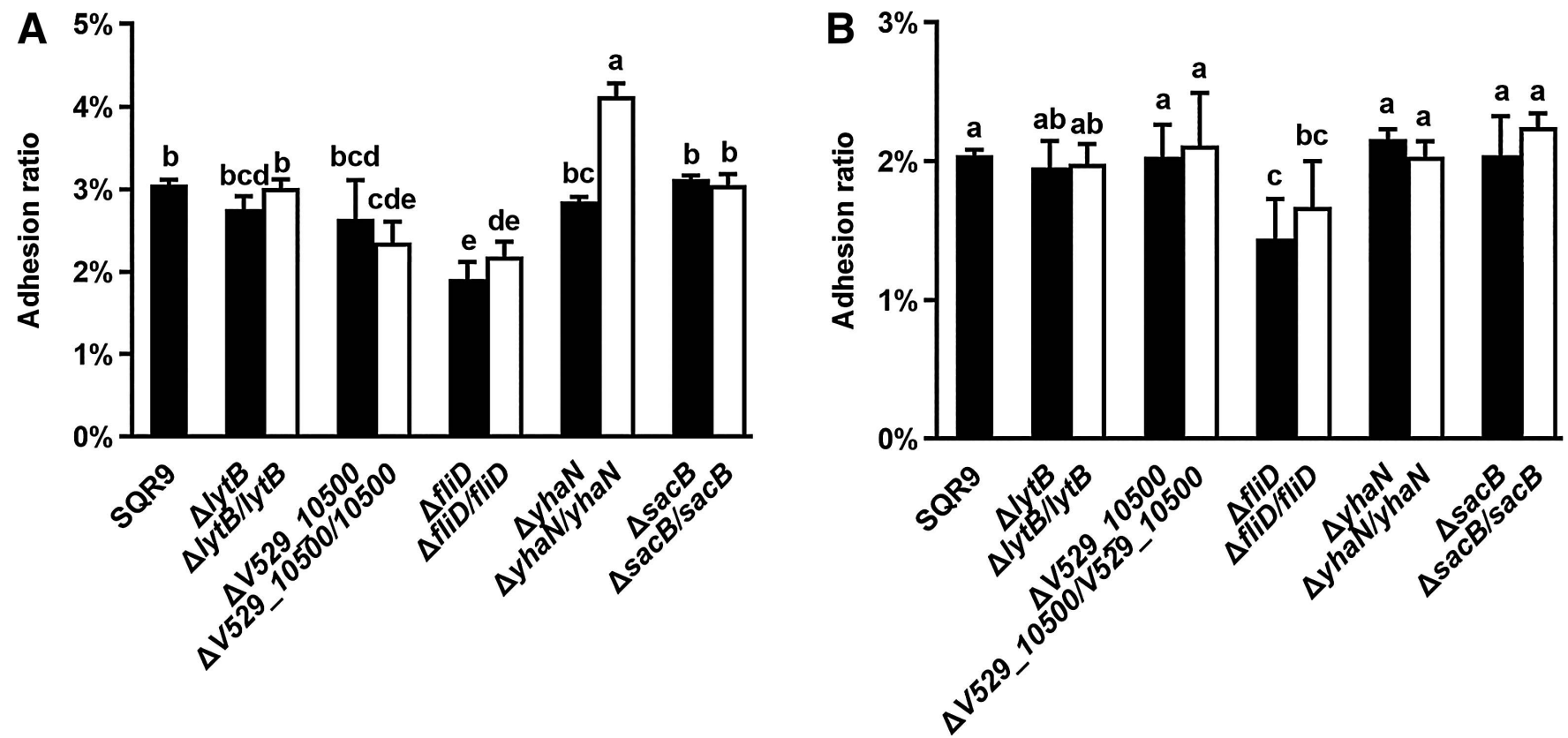

Fig. 3. Adherence ability to abiotic surfaces by the wild-type strain Bacillus velezensis SQR9, adhesin mutants, and complementation strains. The adherence ability was indicated by adhesion ratio, i.e. the number of bacteria attached to abiotic surfaces relative to inoculation amount. A, Adherence to polystyrene surfaces. B, Adherence to glass surfaces. Error bars are standard deviation, $n=4$. Different letters mean significant differences $(P<0.05$, oneway analysis of variance). 
gene in PA Bacillus strains. Thus, fliD might not be conserved in PA Bacillus strains, the same case as for $s a c B$ gene.

\section{DISCUSSION}

Root colonization is a prerequisite for PGPRs to perform their plant beneficial functions; the initial attachment and adhesion are the early stages for root colonization and biofilm formation. In this study, we demonstrated that PGRP strain B. velezensis SQR9 has multiple adhesin proteins for root and abiotic surface attachment, which are required for its efficient rhizoplane colonization.

Gene $f i D$ has great impacts on chemotaxis, adhesion, pellicle biofilm formation, and root colonization, but not the aggregation

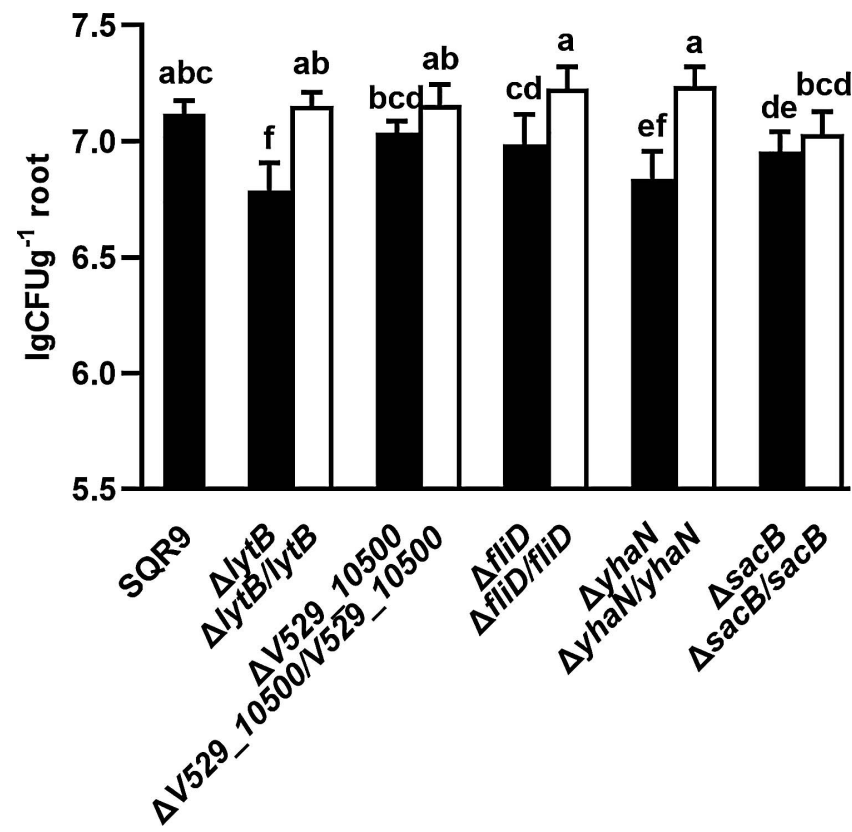

Fig. 4. Colonization of cucumber roots by the wild-type strain Bacillus velezensis SQR9, adhesin mutants, and complementation strains. Cells were diluted in one-fourth Murashige-Skoog medium and the root of a cucumber seedling was immersed into it. After coculturing at room temperature for $24 \mathrm{~h}$, cells colonized on cucumber roots were determined by the plate-counting method. Error bars are standard deviation, $n=5$. Different letters mean significant differences $(P<0.05$, one-way analysis of variance). Results are shown as means of $\mathrm{Lg}$ (number of bacteria cells per gram of fresh root). ability. Mutation of the fliD gene seemed to significantly diminish the movement of B. velezensis SQR9, since $\Delta$ fliD lost the chemotaxis ability. Attachment of B. velezensis SQR9 $\triangle f i D$ mutant to cucumber root and abiotic surfaces were lower than the wild-type strain, which indicated that $f l D$ was involved in the adhesion. This phenomenon can be partially explained by the effect of motility and chemotaxis on the bacterial attachment to both biotic and abiotic surfaces (Van de Broek et al. 1998). Also, in several bacterial species, the flagellar cap protein FliD has been shown to be required for adhesion to surfaces. The FliD protein of Clostridium difficile could bind specifically to mucus isolated from axenic mice (Tasteyre et al. 2001). Mutant $\Delta f i D$ of Pseudomonas aeruginosa cannot bind mucin, but complementation with $f i D$ gene restored the binding phenotype, suggesting that FliD mediates the adhesion to mucin (Arora et al. 1998). In our study, the FliD protein of B. velezensis SQR9 is not only related to bacterial motility but also involved in adhesion. Gene complementation of $\Delta f i D$ did not restore attachment phenotype, which may be due to the complex assembly of flagella. Flagella are complex machines assembled from over 30 different proteins organized into three structural domains, the basal body, the hook, and the filament, and assembly of flagella is strictly time-controlled. Basal bodies are assembled rapidly, followed by the assembly of the hook, then FliD acts as a cap on the end of the extending structure, and finally, the long helical filament is formed through the basal body and assembled underneath the FliD cap (Guttenplan et al. 2013; Mukherjee et al. 2013). Thus, when expressed in situ, FliD and other flagellar proteins are assembled in order, but gene complementation of fiD might not be as timely as in situ expression, which leads to the difficulty to synthesize flagellum filament of the last step and $\Delta$ fliD/fliD cannot restore the attachment phenotype.

It is noticed that the $\Delta y h a N$ mutant complemented with $y h a N$ gene greatly increased adhesion and colonization ability compared with the wild-type strain of B. velezensis SQR9. In SQR9, $y h a N$ and its adjacent genes $y h a P$ and $y h a Q$ are predicted as $\mathrm{ABC}$ transporter genes. The role for $\mathrm{ABC}$ transporters in cell adhesion and biofilm development has been proposed in other organisms. An ABC transporter was important for attachment and virulence of Agrobacterium tumefaciens on carrot cells (Matthysse et al. 1996). In Pseudomonad putida, the potB gene, encoding an $\mathrm{ABC}$ transporter component, was upregulated in the early stages of biofilm formation on silicone (Sauer and Camper 2001). An ABC transporter of Pseudomonas fluorescens WCS365 encoded by lapEBC was predicted to participate in the

Table 2. Identity (\%) between SQR9 and Bacillus amyloliquefaciens plant-associated or B. subtilis non-plant associated strains in adhesin genes or whole genomes

\begin{tabular}{|c|c|c|c|c|c|c|c|c|c|c|c|c|}
\hline \multirow[b]{2}{*}{ Gene $^{\mathbf{a}}$} & \multicolumn{12}{|c|}{ Plant-associated strains $^{b}$} \\
\hline & NJN-6 & LFB112 & Y2 & CAU B946 & NAU-B3 & YAU B9601-Y2 & CC178 & UCMB5033 & UCMB5036 & UCMB5113 & FZB42 & IT-45 \\
\hline lytB & 98 & 98 & 98 & 98 & 98 & 98 & 98 & 98 & 98 & 98 & 98 & 98 \\
\hline fliD & 94 & 94 & 94 & 94 & 95 & 94 & 99 & 94 & 96 & 94 & 99 & 94 \\
\hline$s a c B$ & 98 & 98 & 99 & 98 & 99 & 99 & 99 & 99 & 99 & 99 & 99 & 98 \\
\hline yhaN & 98 & 98 & 99 & 98 & 98 & 99 & 99 & 99 & 99 & 99 & 99 & 98 \\
\hline 10500 & 97 & 97 & 98 & 97 & 98 & 98 & 98 & 98 & 98 & 98 & 98 & 97 \\
\hline \multirow[t]{3}{*}{ SQR9 } & 97 & 97 & 97 & 97 & 97 & 97 & 98 & 98 & 98 & 98 & 98 & 97 \\
\hline & \multicolumn{4}{|c|}{ Non-plant associated strains ${ }^{b}$} & & & & & & & & \\
\hline & DSM7 & LL3 & TA208 & XH7 & & & & & & & & \\
\hline lytB & 67 & 67 & 67 & 67 & & & & & & & & \\
\hline fliD & 92 & 92 & 92 & 92 & & & & & & & & \\
\hline$s a c B$ & 95 & 95 & 95 & 95 & & & & & & & & \\
\hline yhaN & 92 & 92 & 92 & 92 & & & & & & & & \\
\hline 10500 & 84 & 84 & 84 & 84 & & & & & & & & \\
\hline SQR9 & 93 & 93 & 93 & 93 & & & & & & & & \\
\hline
\end{tabular}

a The nucleotide identity (\%) of adhesin genes was calculated using BLAST from the National Center for Biotechnology Information database.

${ }^{\mathrm{b}}$ The whole-genome average nucleotide identity $(\%)$ was calculated by FastANI from KBase. 
secretion of LapA, a large cell-surface protein required for biofilm formation and attachment to quartz sand (Hinsa et al. 2003). These results suggest that the role of $A B C$ transporters in biofilm development may be conserved across organisms, perhaps for the secretion of cell surface adhesins. In SQR9, we postulate that yhaNPQ encode an $\mathrm{ABC}$ transporter participating in the secretion of YhaL, a sporulation protein. Some sporulation proteins might be involved in biofilm formation, for example, comER gene of both B. subtilis and B. cereus; comER gene mutation resulted in defects in biofilm formation and a delay in spore formation in these two Bacillus species (Yan et al. 2016). Therefore, yhaN gene may contribute to adhesion and colonization in an indirect way as an $\mathrm{ABC}$ transporter.

$\mathrm{LytB}$ is known as a peptidoglycan hydrolase, the role of $\mathrm{LytB}$ in Streptococcus pneumonia colonization of the nasopharynx was well-studied (Gosink et al. 2000; Moscoso et al. 2006). Our results showed that $\Delta l y t B$ of $B$. velezensis SQR9 decreased the ability to attach and colonize cucumber root. As a peptidoglycan hydrolase, LytB plays a role in daughter cell separation (García et al. 1999), which might be related to root attachment and colonization.

$\mathrm{SacB}$ is a levansucrase, responsible for the synthesis of levan. Levan activity may contribute to cell aggregation and biofilm maturation. For example, levan acted as binding sites for Streptococcus mutans adhesion (Rozen et al. 2004). Levan also might act as an energy storage for Pseudomonas syringae due to the accumulation of levan in biofilm matrix, implying a function as a nutrient storage source for later stages of biofilm development (Laue et al. 2006). The levansucrase of Gluconacetobacter diazotrophicus is encoded by the $l s d A$ gene, its deficiency decreased the ability to form cell aggregates on abiotic surfaces, and complementation of the mutant with the $l s d A$ gene restored this ability (Velázquez-Hernández et al. 2011). Thus, SacB in $B$. velezensis $\mathrm{SQR} 9$ might contribute to adhesion and colonization ability by adhering to surfaces or forming biofilm structure.

The mutants of $B$. velezensis SQR9 with poor adhesion ability to root surfaces showed a deficiency in colonization on the rhizosphere. Besides, we were interested in the possible correlation between attachment or colonization of $B$. velezensis SQR9 to biotic and to abiotic surfaces and whether the mutants isolated in our screen showed a general deficiency in all surfaces or were just altered in functions specific for attaching to roots and rhizosphere colonization. Since the roles of adhesin genes involved in rhizosphere adhesion are just listed by hypothesis, the detailed analysis of the mechanism of isolated genes of B. velezensis $\mathrm{SQR} 9$ in adhesion needs future studies.

\section{MATERIALS AND METHODS}

\section{Strain and growth condition.}

Bacillus velezensis SQR9 (CGMCC accession number 5808 [China General Microbiology Culture Collection Center]) was cultivated at $37^{\circ} \mathrm{C}$ in $\mathrm{LB}$ medium $(1 \%$ tryptone, $0.5 \% \mathrm{NaCl}$, and $0.3 \%$ yeast extract) solidified with $2 \%$ agar for routine growth (Cao et al. 2011)

\section{Construction of mutants and complementation strains.}

Mutants of $B$. velezensis SQR9 deficient in LytB, V529_ 10500, YcdA, V529_07150, FliD, YhaN, or SacB were constructed with marker-free strategy as described previously (Zhou et al. 2017). To complement the deleted genes in these mutants, the $a m y E$ gene was used as an insertion site, and $100 \mathrm{mg}$ of spectinomycin per milliliter was used to select the gene complementation strains. Plasmid pNW33N containing the green fluorescent protein ( $g f p$ ) gene (Zhou et al. 2018) was transformed to construct GFP-tagged strains. All these mutants and gene complementation stains are listed in Supplementary Table S1.

\section{Plant growth.}

Cucumber (Cucumis sativus L. variety Jinchun 4) was used in this study. The seeds of cucumber were surface-sterilized in $2 \%$ $\mathrm{NaClO}$ for $8 \mathrm{~min}$ and in $75 \%$ ethanol for $30 \mathrm{~s}$ and were then washed by distilled water three times. The seeds were germinated on a sterilized wet filter paper in a Petri dish and were grown at $30^{\circ} \mathrm{C}$ in the dark until the radicle reached 1.5 to 2.0 $\mathrm{cm}$. Next, the seedlings were moved out, were washed, and were placed into 50-ml flasks, containing one-fourth MurashigeSkoog medium, for growth at $30^{\circ} \mathrm{C}$ under $16 \mathrm{~h}$ of illumination with regularly changing of solution.

\section{Chemotaxis assays.}

Chemotaxis was tested with a drop assay (Grimm and Harwood 1997). Bacterial cells in the logarithmic phase of growth were harvested and were resuspended in an equal volume of chemotaxis buffer. Aqueous solution of $1 \%$ hydroxypropyl methylcellulose was added to the cell suspension to restrict the spread of the chemoattractant. The viscous cell suspension was then layered on the bottoms of 60 -mm-diameter Petri dishes, to a depth of about $3 \mathrm{~mm}$, and $10 \mu \mathrm{l}$ of $100 \mathrm{mM}$ malic acid was added to the center of a dish as an attractant. The chemotactic response of cells to the added compound resulted in the formation of a turbid ring near the center of the Petri dish after $10 \mathrm{~min}$. Three replications of this experiment were conducted.

\section{Adherence to cucumber roots.}

The root attachment assay was described by Smit (1986) with modification. Bacterial cultures were centrifuged at $4,000 \times g$ for $5 \mathrm{~min}$, the pellets were washed three times with phosphate buffer (PBS) (pH 7.5) and were suspended to a final optical density at $600 \mathrm{~nm}\left(\mathrm{OD}_{600}\right)$ of 0.002 , which corresponds to $5 \times 10^{5}$ bacterial cells per milliliter (Supplementary Fig. S9A), the number of cells was analyzed by the plate counting method. This bacterial suspension was used to immerse roots. Young cucumber lateral roots were chosen to be cut into segments approximately $3 \mathrm{~cm}$ long. These segments were immersed in $10 \mathrm{ml}$ of bacterial suspension for $30 \mathrm{~min}$ at room temperature in static after mixing. Then, root segments were washed eight times in PBS to remove free and weakly attached bacteria. After sonication for $30 \mathrm{~s}$ and vortexing until the adhered bacteria were detached from roots, the number of attached bacterial cells was analyzed by the plate counting method. Five replications of this experiment were conducted.

\section{Adherence to polystyrene or glass surfaces.}

Bacterial cultures were centrifuged at $4,000 \times g$ for $5 \mathrm{~min}$. The pellet was washed three times with PBS and was suspended in the same solution. The cells were diluted to a final $\mathrm{OD}_{600}$ of 0.0004 in PBS in a well of a 48-well polystyrene plate, corresponding to $10^{5}$ bacterial cells per milliliter (Supplementary Fig. S9B). The cells were diluted to a final $\mathrm{OD}_{600}$ of 0.004 in PBS in a glass tube, corresponding to $10^{6}$ bacterial cells per milliliter (Supplementary Fig. S9C). The number of inoculation cells was analyzed by the plate counting method. The bacterial suspension was then injected into a plate or glass tube for $30 \mathrm{~min}$ at room temperature in static after mixing. Then, bacterial cultures in wells or tubes were discarded. Wells or tubes were gently washed five times in PBS to remove free and weakly attached bacteria. Attached cells were suspended in the sterile PBS. After sonication for $30 \mathrm{~s}$ and vortexing until the adhered bacteria were detached from wells or tubes, attached cells were counted by the plate counting method. Four replications of this experiment were conducted.

\section{Adhesion observation by fluorescent microscopy.}

Sterilized cucumber roots were prepared as mentioned above, the capillary and plastic filament were chosen as glass and plastic material, respectively. GFP-tagged strains were grown in LB 
medium containing $5 \mathrm{mg}$ of chloramphenicol per milliliter for $12 \mathrm{~h}$. The bacterial cultures of different strains were harvested, were washed with PBS, and were suspended to the same concentration. Roots, capillaries, and plastic filaments were cut into segments approximately $3 \mathrm{~cm}$ long. These segments were immersed in $10 \mathrm{ml}$ of bacterial suspension for $30 \mathrm{~min}$ at room temperature in static. Then, segments were washed in PBS to remove free and weakly attached bacteria and the segments were observed immediately by CLSM (Leica Model TCS SP2; Heidelberg, Germany). Excitation wavelengths of $488 \mathrm{~nm}$ and emitted wavelengths of 500 to $600 \mathrm{~nm}$ were collected for the GFP. Three replications of this experiment were conducted.

\section{Aggregation and pellicle biofilm formation.}

For aggregation ability analysis, strains were inoculated on solid MSgg medium ( $5 \mathrm{mM}$ potassium phosphate, $100 \mathrm{mM}$ morpholine propanesulfonic acid, $2 \mathrm{mM} \mathrm{MgCl} 2,700 \mathrm{M} \mathrm{CaCl}_{2}, 50 \mathrm{M}$ $\mathrm{MnCl}_{2}, 50 \mathrm{M} \mathrm{FeCl}_{3}, 1 \mathrm{M} \mathrm{ZnCl}_{2}, 2 \mathrm{mM}$ thiamine, $0.5 \%$ glycerol, $0.5 \%$ glutamate, $50 \mathrm{~g}$ of tryptophan per milliliter, $50 \mathrm{~g}$ of phenylalanine per milliliter, and $50 \mathrm{~g}$ of threonineper milliliter) and were cultured at $37^{\circ} \mathrm{C}$ for $12 \mathrm{~h}$, and aggregation architectures were recorded by the stereoscope. For colony formation, each strain was grown overnight in LB broth, and a 3- $\mu$ l sample of the culture was spotted onto LB agar and was incubated at $37^{\circ} \mathrm{C}$. To assess pellicle biofilm formation, $10 \mu \mathrm{l}$ of suspension was inoculated into standing culture consisting of $1 \mathrm{ml}$ of MSgg in a microtiter plate well, and the cultures were incubated at $37^{\circ} \mathrm{C}$ without shaking. Three replications of each experiment were included.

\section{Colonization on the rhizosphere.}

Bacterial suspension was inoculated into one-fourth Murashige-Skoog medium to make the final $\mathrm{OD}_{600}$ value of 0.1 , into which sterile cucumber seedlings with three true leaves were immersed. After slowly shaking, cells colonized on cucumber roots were determined by plate colony counting $24 \mathrm{~h}$ after inoculation at room temperature. In detail, roots were washed eight times in PBS to remove free and weakly attached bacteria. After vortexing for $5 \mathrm{~min}$ until colonized bacteria were detached from roots, $100 \mu \mathrm{l}$ of the bacterial suspension was analyzed by the plate counting method and were incubated at $37^{\circ} \mathrm{C}$ for $24 \mathrm{~h}$. Five replications of this experiment were included.

\section{Colonization on polystyrene or glass surfaces.}

For the colonization assays, bacterial cells were cultured in $3 \mathrm{ml}$ of $\mathrm{LB}$, containing an appropriate antibiotic, at $37^{\circ} \mathrm{C}$ with shaking. When $\mathrm{OD}_{600}$ reached 1 , the cells were diluted to $1: 10$ in one-fourth Murashige-Skoog medium in a 48-well polystyrene plate or glass tube and were incubated at $37^{\circ} \mathrm{C}$. The colonization ability was determined after $24 \mathrm{~h}$ by the crystal violet staining method. In detail, pellicles in wells or tubes were gently removed with PBS before drying up, biofilms that adhered to wells or tubes were stained with $1 \mathrm{ml} 0.1 \%$ crystal violet for 30 min and were washed three times with PBS. Next, $1 \mathrm{ml}$ of $70 \%$ ethanol was added to dissolve the biofilm for $20 \mathrm{~min}$ at room temperature before measuring the $\mathrm{OD}_{570}$ values. Three replications of this experiment were included.

\section{Analysis of nucleotide sequence identity (\%).}

Sixteen strains of $B$. amyloliquefaciens or $B$. velezensis (Zhang et al. 2016) have been chosen, 12 PA strains and four non-PA. They were compared with SQR9 for these five adhesin genes and for the whole genome. Nucleotide BLAST from NCBI was used for comparisons of adhesin genes in PA and non-PA Bacillus strains. In detail, FASTA sequence of each SQR9 adhesin gene was input as query sequence and the accession number of each $B$. amyloliquefaciens or $B$. subtilis strain was input as subject sequence. The results have been optimized for "somewhat similar sequences". FastANI from KBase was used for ANI (\%) of the whole genome of SQR9 and PA and non-PA B. amyloliquefaciens and B. subtilis, respectively, strains. FASTA sequence of the whole genome of each strain was uploaded to this website for FastANI results between SQR9 and other B. amyloliquefaciens/B. subtilis strains.

\section{AUTHOR-RECOMMENDED INTERNET RESOURCE}

KBase: https://narrative.kbase.us/narratives

\section{LITERATURE CITED}

Aguilar, C., Vlamakis, H., Losick, R., and Kolter, R. 2007. Thinking about Bacillus subtilis as a multicellular organism. Curr. Opin. Microbiol. 10:638-643.

Arora, S. K., Ritchings, B. W., Almira, E. C., Lory, S., and Ramphal, R. 1998. The Pseudomonas aeruginosa flagellar cap protein, FliD, is responsible for mucin adhesion. Infect. Immun. 66:1000-1007.

Balsanelli, E., Serrato, R. V., de Baura, V. A., Sassaki, G., Yates, M. G., Rigo, L. U., Pedrosa, F. O., de Souza, E. M., and Monteiro, R. A. 2010. Herbaspirillum seropedicae $r f b B$ and $r f b C$ genes are required for maize colonization. Environ. Microbiol. 12:2233-2244.

Bökel, C., and Brown, N. H. 2002. Integrins in development: Moving on, responding to, and sticking to the extracellular matrix. Dev. Cell 3: 311-321.

Bowen, W. R., Fenton, A. S., Lovitt, R. W., and Wright, C. J. 2002. The measurement of Bacillus mycoides spore adhesion using atomic force microscopy, simple counting methods, and a spinning disk technique. Biotechnol. Bioeng. 79:170-179.

Cao, Y., Zhang, Z., Ling, N., Yuan, Y., Zheng, X., Shen, B., and Shen, Q. 2011. Bacillus subtilis SQR9 can control Fusarium wilt in cucumber by colonizing plant roots. Biol. Fertil. Soils 47:495-506.

Crawford, R. W., Reeve, K. E., and Gunn, J. S. 2010. Flagellated but not hyperfimbriated Salmonella enterica serovar Typhimurium attaches to and forms biofilms on cholesterol-coated surfaces. J. Bacteriol. 192: 2981-2990.

Croes, C. L., Moens, S., Vanbastelaere, E., Vanderleyden, J., and Michiels, K. W. 1993. The polar flagellum mediates Azospirillum brasilense adsorption to wheat roots. J. Gen. Appl. Microbiol. 139:2261-2269.

de Souza, A. A., Takita, M. A., Pereira, E. O., Coletta-Filho, H. D., and Machado, M. A. 2005. Expression of pathogenicity-related genes of Xylella fastidiosa in vitro and in planta. Curr. Microbiol. 50:223-228.

Dickens, N. J., Beatson, S., and Ponting, C. P. 2002. Cadherin-like domains in alpha-dystroglycan, alpha/epsilon-sarcoglycan and yeast and bacterial proteins. Curr. Biol. 12:R197-R199.

Dong, X., Liu, Y., Zhang, G., Wang, D., Zhou, X., Shao, J., Shen, Q., and Zhang, R. 2018. Synthesis and detoxification of nitric oxide in the plant beneficial rhizobacterium Bacillus amyloliquefaciens SQR9 and its effect on biofilm formation. Biochem. Biophys. Res. Commun. 503:784-790.

Faille, C., Lequette, Y., Ronse, A., Slomianny, C., Garénaux, E., and Guerardel, Y. 2010. Morphology and physico-chemical properties of Bacillus spores surrounded or not with an exosporium: Consequences on their ability to adhere to stainless steel. Int. J. Food Microbiol. 143: 125-135.

Fan, B., Chen, X. H., Budiharjo, A., Bleiss, W., Vater, J., and Borriss, R. 2011. Efficient colonization of plant roots by the plant growth promoting bacterium Bacillus amyloliquefaciens FZB42, engineered to express green fluorescent protein. J. Biotechnol. 151:303-311.

Feng, H., Zhang, N., Du, W., Zhang, H., Liu, Y., Fu, R., Shao, J., Zhang, G., Shen, Q., and Zhang, R. 2018. Identification of chemotaxis compounds in root exudates and their sensing chemoreceptors in plantgrowth-promoting rhizobacteria Bacillus amyloliquefaciens SQR9. Mol. Plant-Microbe Interact 31:995-1005.

García, P., González, M. P., García, E., López, R., and García, J. L. 1999. LytB, a novel pneumococcal murein hydrolase essential for cell separation. Mol. Microbiol. 31:1275-1281.

Genevaux, P., Bauda, P., DuBow, M. S., and Oudega, B. 1999. Identification of Tn10 insertions in the $r f a G, r f a P$, and galU genes involved in lipopolysaccharide core biosynthesis that affect Escherichia coli adhesion. Arch. Microbiol. 172:1-8.

Gopalani, M., Dhiman, A., Rahi, A., and Bhatnagar, R. 2016. Overexpression of the pleiotropic regulator CodY decreases sporulation, attachment and pellicle formation in Bacillus anthracis. Biochem. Biophys. Res. Commun. 469:672-678. 
Gosink, K. K., Mann, E. R., Guglielmo, C., Tuomanen, E. I., and Masure, H. R. 2000. Role of novel choline binding proteins in virulence of Streptococcus pneumoniae. Infect. Immun. 68:5690-5695.

Grimm, A. C., and Harwood, C. S. 1997. Chemotaxis of Pseudomonas spp. to the polyaromatic hydrocarbon naphthalene. Appl. Environ. Microbiol. 63:4111-4115.

Guttenplan, S. B., Shaw, S., and Kearns, D. B. 2013. The cell biology of peritrichous flagella in Bacillus subtilis. Mol. Microbiol. 87:211-229.

Hall-Stoodley, L., Costerton, J. W., and Stoodley, P. 2004. Bacterial biofilms: From the natural environment to infectious diseases. Nat. Rev. Microbiol. 2:95-108.

Hinsa, S. M., Espinosa-Urgel, M., Ramos, J. L., and O'Toole, G. A 2003. Transition from reversible to irreversible attachment during biofilm formation by Pseudomonas fluorescens WCS365 requires an ABC transporter and a large secreted protein. Mol. Microbiol. 49:905-918.

Hori, K., and Matsumoto, S. 2010. Bacterial adhesion: From mechanism to control. Biochem. Eng. J. 48:424-434

Kelly, G., Prasannan, S., Daniell, S., Fleming, K., Frankel, G., Dougan, G., Connerton, I., and Matthews, S. 1999. Structure of the celladhesion fragment of intimin from enteropathogenic Escherichia coli. Nat. Struct. Biol. 6:313-318.

Kesel, S., Mader, A., Seeberger, P. H., Lieleg, O., and Opitz, M. 2014. Carbohydrate coating reduces adhesion of biofilm-forming Bacillus subtilis to gold surfaces. Appl. Environ. Microbiol. 80:5911-5917.

Klavenes, A., Stalheim, T., Sjovold, O., Josefsen, K., and Granum, P. E. 2002. Attachment of Bacillus cereus spores with and without appendages to stainless steel surfaces. Food Bioprod. Process. 80:312-318.

Knoke, L. R., Abad Herrera, S., Götz, K., Justesen, B. H., Günther Pomorski, T., Fritz, C., Schäkermann, S., Bandow, J. E., and Aktas, M. 2020. Agrobacterium tumefaciens small lipoprotein Atu8019 is involved in selective outer membrane vesicle (OMV) docking to bacterial cells. Front. Microbiol. 11:1228.

Laue, H., Schenk, A., Li, H., Lambertsen, L., Neu, T. R., Molin, S., and Ullrich, M. S. 2006. Contribution of alginate and levan production to biofilm formation by Pseudomonas syringae. Microbiol. Read. 152: 2909-2918.

Laus, M. C., Logman, T. J., Lamers, G. E., Van Brussel, A. A. N., Carlson, R. W., and Kijne, J. W. 2006. A novel polar surface polysaccharide from Rhizobium leguminosarum binds host plant lectin. Mol. Microbiol. 59:1704-1713.

Laus, M. C., Logman, T. J., Van Brussel, A. A. N., Carlson, R. W., Azadi, P., Gao, M. Y., and Kijne, J. W. 2004. Involvement of exo5 in production of surface polysaccharides in Rhizobium leguminosarum and its role in nodulation of Vicia sativa subsp. nigra. J. Bacteriol. 186:6617-6625.

Lazarevic, V., Margot, P., Soldo, B., and Karamata, D. 1992. Sequencing and analysis of the Bacillus subtilis lyt $R A B C$ divergon: A regulatory unit encompassing the structural genes of the $N$-acetylmuramoyl-L-alanine amidase and its modifier. J. Gen. Microbiol. 138:1949-1961.

Lequette, Y., Garénaux, E., Tauveron, G., Dumez, S., Perchat, S., Slomianny, C., Lereclus, D., Guérardel, Y., and Faille, C. 2011. Role played by exosporium glycoproteins in the surface properties of Bacillus cereus spores and in their adhesion to stainless steel. Appl. Environ. Microbiol. 77:4905-4911.

Li, Q., Li, Z., Li, X., Xia, L., Zhou, X., Xu, Z., Shao, J., Shen, Q., and Zhang, R. 2018. FtsEX-CwlO regulates biofilm formation by a plantbeneficial rhizobacterium Bacillus velezensis SQR9. Res. Microbiol. 169:166-176.

López, R., and García, E. 2004. Recent trends on the molecular biology of pneumococcal capsules, lytic enzymes, and bacteriophage. FEMS Microbiol. Rev. 28:553-580.

Makin, S. A., and Beveridge, T. J. 1996. The influence of A-band and B-band lipopolysaccharide on the surface characteristics and adhesion of Pseudomonas aeruginosa to surfaces. Microbiol. Read. 142:299-307.

Manandhar, S., Vidhate, S., and D'Souza, N. 2009. Water soluble levan polysaccharide biopolymer electrospun fibers. Carbohydr. Polym. 78: 794-798.

Matthysse, A. G., Yarnall, H. A., and Young, N. 1996. Requirement for genes with homology to $\mathrm{ABC}$ transport systems for attachment and virulence of Agrobacterium tumefaciens. J. Bacteriol. 178:5302-5308.

Merritt, P. M., Danhorn, T., and Fuqua, C. 2007. Motility and chemotaxis in Agrobacterium tumefaciens surface attachment and biofilm formation. J. Bacteriol. 189:8005-8014.

Mistry, J., Chuguransky, S., Williams, L., Qureshi, M., Salazar, G. A., Sonnhammer, E. L. L., Tosatto, S. C. E., Paladin, L., Raj, S., Richardson, L. J., Finn, R. D., and Bateman, A. 2021. Pfam: The protein families database in 2021. Nucleic Acids Res. 49 (D1):D412-D419.
Moreira, L. M., de Souza, R. F., Almeida, N. F., Jr., Setubal, J. C. Oliveira, J. C. F., Furlan, L. R., Ferro, J. A., and da Silva, A. C. R. 2004. Comparative genomics analyses of citrus-associated bacteria. Annu. Rev. Phytopathol. 42:163-184.

Moscoso, M., García, E., and López, R. 2006. Biofilm formation by Streptococcus pneumoniae: Role of choline, extracellular DNA, and capsular polysaccharide in microbial accretion. J. Bacteriol. 188: 7785-7795.

Mukherjee, S., Babitzke, P., and Kearns, D. B. 2013. FliW and FliS function independently to control cytoplasmic flagellin levels in Bacillus subtilis. J. Bacteriol. 195:297-306.

Nevola, J. J., Stocker, B. A. D., Laux, D. C., and Cohen, P. S. 1985. Colonization of the mouse intestine by an avirulent Salmonella typhimurium strain and its lipopolysaccharide-defective mutants. Infect. Immun. 50:152-159.

Parkar, S. G., Flint, S. H., Palmer, J. S., and Brooks, J. D. 2001. Factors influencing attachment of thermophilic bacilli to stainless steel. J. Appl. Microbiol. 90:901-908.

Pereira, Y., Petit-Glatron, M. F., and Chambert, R. 2001. yveB, encoding endolevanase LevB, is part of the $s a c B-y v e B-y v e A$ levansucrase tricistronic operon in Bacillus subtilis. Microbiol. Read. 147:3413-3419.

Pratt, L. A., and Kolter, R. 1998. Genetic analysis of Escherichia coli biofilm formation: Roles of flagella, motility, chemotaxis and type I pili. Mol. Microbiol. 30:285-293.

Qiu, M., Li, S., Zhou, X., Cui, X., Vivanco, J. M., Zhang, N., Shen, Q., and Zhang, R. 2014. De-coupling of root-microbiome associations followed by antagonist inoculation improves rhizosphere soil suppressiveness. Biol. Fertil. Soils 50:217-224.

Ramey, B. E., Koutsoudis, M., von Bodman, S. B., and Fuqua, C. 2004. Biofilm formation in plant-microbe associations. Curr. Opin. Microbiol. 7:602-609.

Ramos-Sevillano, E., Moscoso, M., García, P., García, E., and Yuste, J. 2011. Nasopharyngeal colonization and invasive disease are enhanced by the cell wall hydrolases LytB and LytC of Streptococcus pneumoniae. PLoS One 6:e23626.

Rozen, R., Bachrach, G., and Steinberg, D. 2004. Effect of carbohydrates on fructosyltransferase expression and distribution in Streptococcus mutans GS-5 biofilms. Carbohydr. Res. 339:2883-2888.

Sadiq, F. A., Flint, S., Sakandar, H. A., and He, G. 2019. Molecular regulation of adhesion and biofilm formation in high and low biofilm producers of Bacillus licheniformis using RNA-Seq. Biofouling 35:143-158.

Sánchez, B., Arias, S., Chaignepain, S., Denayrolles, M., Schmitter, J. M., Bressollier, P., and Urdaci, M. C. 2009. Identification of surface proteins involved in the adhesion of a probiotic Bacillus cereus strain to mucin and fibronectin. Microbiol. Read. 155:1708-1716.

Sauer, K., and Camper, A. K. 2001. Characterization of phenotypic changes in Pseudomonas putida in response to surface-associated growth. J. Bacteriol. 183:6579-6589.

Smit, G., Kijne, J. W., and Lugtenberg, B. J. J. 1986. Correlation between extracellular fibrils and attachment of Rhizobium leguminosarum to pea root hair tips. J. Bacteriol. 168:821-827.

Smit, G., Logman, T. J. J., Boerrigter, M. E. T. I., Kijne, J. W., and Lugtenberg, B. J. J. 1989. Purification and partial characterization of the Rhizobium leguminosarum biovar viciae $\mathrm{Ca}^{2+}$-dependent adhesin, which mediates the first step in attachment of cells of the family Rhizobiaceae to plant root hair tips. J. Bacteriol. 171:4054-4062.

Stewart, P. S., and Franklin, M. J. 2008. Physiological heterogeneity in biofilms. Nat. Rev. Microbiol. 6:199-210.

Symersky, J., Patti, J. M., Carson, M., House-Pompeo, K., Teale, M., Moore, D., Jin, L., Schneider, A., DeLucas, L. J., Höök, M., and Narayana, S. V. 1997. Structure of the collagen-binding domain from a Staphylococcus aureus adhesin. Nat. Struct. Biol. 4:833-838.

Tanaka, K. 2000. Formin family proteins in cytoskeletal control. Biochem. Biophys. Res. Commun. 267:479-481.

Tasteyre, A., Barc, M. C., Collignon, A., Boureau, H., and Karjalainen, T. 2001. Role of FliC and FliD flagellar proteins of Clostridium difficile in adherence and gut colonization. Infect. Immun. 69:7937-7940.

Tonry, J. H., McNichol, B. A., Ramarao, N., Chertow, D. S., Kim, K. S., Stibitz, S., Schneewind, O., Kashanchi, F., Bailey, C. L., Popov, S., and Chung, M. C. 2012. Bacillus anthracis protease InhA regulates BslA-mediated adhesion in human endothelial cells. Cell. Microbiol. 14:1219-1230.

Tran, S. L., Guillemet, E., Gohar, M., Lereclus, D., and Ramarao, N. 2010. CwpFM (EntFM) is a Bacillus cereus potential cell wall peptidase implicated in adhesion, biofilm formation, and virulence. J. Bacteriol. 192:2638-2642. 
Van de Broek, A., Lambrecht, M., and Vanderleyden, J. 1998. Bacterial chemotactic motility is important for the initiation of wheat root colonization by Azospirillum brasilense. Microbiol. Read. 144:2599-2606.

Velázquez-Hernández, M. L., Baizabal-Aguirre, V. M., Cruz-Vázquez, F., Trejo-Contreras, M. J., Fuentes-Ramírez, L. E., Bravo-Patiño, A., Cajero-Juárez, M., Chávez-Moctezuma, M. P., and Valdez-Alarcón, J. J. 2011. Gluconacetobacter diazotrophicus levansucrase is involved in tolerance to $\mathrm{NaCl}$, sucrose and desiccation, and in biofilm formation. Arch. Microbiol. 193:137-149.

Vlamakis, H., Chai, Y., Beauregard, P., Losick, R., and Kolter, R. 2013. Sticking together: Building a biofilm the Bacillus subtilis way. Nat. Rev. Microbiol. 11:157-168.

Walton, T. A., and Sousa, M. C. 2004. Crystal structure of Skp, a prefoldin-like chaperone that protects soluble and membrane proteins from aggregation. Mol. Cell 15:367-374.

Weng, J., Wang, Y., Li, J., Shen, Q., and Zhang, R. 2013. Enhanced root colonization and biocontrol activity of Bacillus amyloliquefaciens SQR9 by abrB gene disruption. Appl. Microbiol. Biotechnol. 97:8823-8830.

Xu, Z., Mandic-Mulec, I., Zhang, H., Liu, Y., Sun, X., Feng, H., Xun, W., Zhang, N., Shen, Q., and Zhang, R. 2019. Antibiotic bacillomycin $\mathrm{D}$ affects iron acquisition and biofilm formation in Bacillus velezensis through a Btr-mediated FeuABC-dependent pathway. Cell Rep. 29: 1192-1202.e5.

Xu, Z., Shao, J., Li, B., Yan, X., Shen, Q., and Zhang, R. 2013. Contribution of bacillomycin D in Bacillus amyloliquefaciens SQR9 to antifungal activity and biofilm formation. Appl. Environ. Microbiol. 79:808-815.
Xu, Z., Zhang, R., Wang, D., Qiu, M., Feng, H., Zhang, N., and Shen, Q. 2014. Enhanced control of cucumber wilt disease by Bacillus amyloliquefaciens SQR9 by altering the regulation of Its DegU phosphorylation. Appl. Environ. Microbiol. 80:2941-2950.

Yan, F., Yu, Y., Wang, L., Luo, Y., Guo, J. H., and Chai, Y. 2016. The comER gene plays an important role in biofilm formation and sporulation in both Bacillus subtilis and Bacillus cereus. Front. Microbiol. 7:1025.

Zhang, N., Yang, D., Kendall, J. R. A., Borriss, R., Druzhinina, I. S., Kubicek, C. P., Shen, Q., and Zhang, R. 2016. Comparative genomic analysis of Bacillus amyloliquefaciens and Bacillus subtilis reveals evolutional traits for adaptation to plant-associated habitats. Front. Microbiol. 7:2039.

Zhao, X., Wang, Y., Shang, Q., Li, Y., Hao, H., Zhang, Y., Guo, Z. Yang, G., Xie, Z., and Wang, R. 2015. Collagen-like proteins (ClpA, $\mathrm{ClpB}$, ClpC, and $\mathrm{ClpD}$ ) are required for biofilm formation and adhesion to plant roots by Bacillus amyloliquefaciens FZB42. PLoS One 10:e0117414.

Zhou, C., Shi, L., Ye, B., Feng, H., Zhang, J., Zhang, R., and Yan, X. 2017. phe $S^{*}$, an effective host-genotype-independent counter-selectable marker for marker-free chromosome deletion in Bacillus amyloliquefaciens. Appl. Microbiol. Biotechnol. 101:217-227.

Zhou, X., Zhang, N., Xia, L., Li, Q., Shao, J., Shen, Q., and Zhang, R. 2018. ResDE two-component regulatory system mediates oxygen limitation-induced biofilm formation in Bacillus amyloliquefaciens SQR9. Appl. Environ. Microbiol. 84:e02744-17. 